\title{
Investigating the evolution of merger remnants from the formation of gas disks
}

\author{
Junko Ueda ${ }^{1,2,3}$ Daisuke Iono $^{1,4}$, Min S. Yun ${ }^{5}$, Alison F. Crocker ${ }^{6}$, \\ Desika Narayanan $^{7}$, Shinya Komugi ${ }^{1}$, Daniel Espada ${ }^{1,8}$, Bunyo \\ Hatsukade $^{1}$, Hiroyuki Kaneko ${ }^{9}$, Yuichi Matsuda ${ }^{1}$, Yoichi Tamura ${ }^{10}$, \\ David J. Wilner ${ }^{3}$, Ryohei Kawabe ${ }^{1,2,4}$ and Hsi-An Pan ${ }^{4,11,12}$
}

${ }^{1}$ National Astronomical Observatory of Japan, 2-21-1 Osawa, Mitaka,Tokyo, 181-8588, Japan email: junko.ueda@nao.ac.jp

${ }^{2}$ Department of Astronomy, School of Science, The University of Tokyo,

7-3-1 Hongo, Bunkyo-ku, Tokyo 133-0033, Japan

${ }^{3}$ Harvard-Smithsonian Center for Astrophysics, 60 Garden Street, Cambridge, MA 02138, USA

${ }^{4}$ Department of Astronomical Science, The Graduate University for Advanced Studie

(SOKENDAI), 2-21-1 Osawa, Mitaka, Tokyo 181-8588, Japan

${ }^{5}$ Department of Astronomy, University of Massachusetts, Amherst, MA 01003, USA

${ }^{6}$ Ritter Astrophysical Research Center, University of Toledo, Toledo, OH 43606, USA

${ }^{7}$ Department of Astronomy, Haverford College, 370 Lancaster Ave, Haverford, PA 19041, USA

${ }^{8}$ Joint ALMA Observatory, Alonso de Córdova 3107, Vitacura, Santiago 763-0355, Chile

${ }^{9}$ Graduate School of Pure and Applied Sciences, University of Tsukuba, 1-1-1 Tennodai, Tsukuba, Ibaraki 305-8577, Japan

${ }^{10}$ IoA, The University of Tokyo, 2-21-1 Osawa, Mitaka,Tokyo, 181-0015, Japan

${ }^{11}$ Nobeyama Radio Observatory, 462-2 Minamimaki, Minamisaku, Nagano 384-1305, Japan

${ }^{12}$ Dept. of Physics, Faculty of Science, Hokkaido Univ., Kita-ku, Sapporo 060-0810, Japan

\begin{abstract}
Our new compilation of interferometric CO data suggests that nuclear and extended molecular gas disks are common in the final stages of mergers. Comparing the sizes of the molecular gas disk and gas mass fractions to early-type and late-type galaxies, about half of the sample show similar properties to early-type galaxies, which have compact gas disks and low gas mass fractions. We also find that sources with extended gas disks and large gas mass fractions may become disk-dominated galaxies.
\end{abstract}

Keywords. galaxies: evolution - galaxies: formation - galaxies: interactions - galaxies: ISM galaxies: kinematics and dynamics - radio lines: galaxies

Major mergers of two disk galaxies are widely believed to provide a way to form a spheroid-dominated early-type galaxy (ETG; e.g., Barnes \& Hernquist 1992). Contrary to the classical scenario, recent simulations with more realistic gas physics have shown that some mergers will reform extended gas disks and evolve into disk-dominated latetype galaxies (LTGs; e.g., Springel \& Hernquist 2005). In order to check this scenario, we investigate interferometric CO maps of 37 optically-selected merger remnants, which are taken from the merger remnant sample (Rothberg \& Joseph 2004). New maps were obtained toward 27/37 sources with ALMA, CARMA, and SMA, and the CO detection rate is $74 \%(20 / 27)$. We find that $80 \%(24 / 30)$ of the sources with robust CO detections show kinematical signatures of the molecular gas disk in their velocity fields (Figure 1).

We compare the properties of molecular gas and stellar components in the merger remnants with ETGs and LTGs to investigate the evolution of merger remnants. The majority of the merger remnants shows a compact molecular gas disk relative to the stellar component. Unless the disks grow significantly, for example from the return of ejected molecular gas or tidal HI gas, the sources will likely evolve into ETGs. We tentatively 


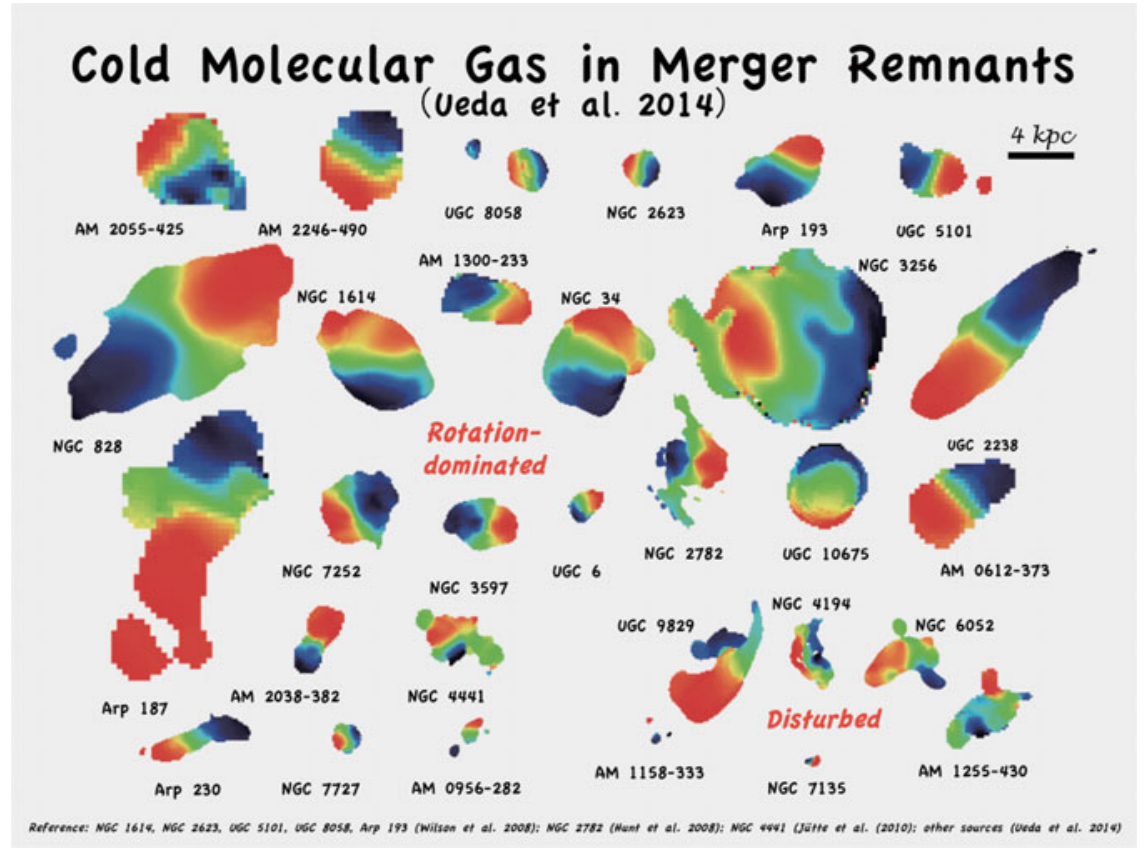

Figure 1. The CO velocity fields of 30 merger remnants (References of the CO data: Hunt et al. 2008; Wilson et al. 2008; Jütte et al. 2010; Ueda et al. 2014). The CO data products are publicly released on the website (http://alma-intweb.mtk.nao.ac.jp/ jueda/data/mr/data.html).

suggest that sources with extended gas disks and large gas mass fractions may become disk-dominated LTGs, if there are no further mechanisms to transport the molecular gas toward the central region thereby decreasing the disk size.

\section{Acknowledgements}

We thank L. K. Hunt, S. García-Burillo, E. Juette, B. Rothberg, and C. D. Wilson for kindly providing their published data. This paper has made use of the following ALMA data: ADS/JAO.ALMA\#2011.0.00099.S and ALMA Science Verification data: ADS/JAO.ALMA\#2011.0.00002.SV. ALMA is a partnership of ESO (representing its member states), NSF (USA) and NINS (Japan), together with NRC (Canada) and NSC and ASIAA (Taiwan), in cooperation with the Republic of Chile. The Joint ALMA Observatory is operated by ESO, AUI/NRAO and NAOJ.

This research was supported, in part, by a grant from the Hayakawa Satio Fund awarded by the Astronomical Society of Japan. J. U. is financially supported by a Research Fellowship from the Japan Society for the Promotion of Science for Young Scientists.

\section{References}

Barnes, J. E. \& Hernquist, L. 1992, ARA\&A, 30, 705

Hunt, L. K., Combes, F., García-Burillo, S., et al. 2008, A\&A, 482, 133

Jütte, E., Aalto, S., \& Hüttemeister, S. 2010, A\& A, 509, A19

Rothberg, B. \& Joseph, R. D. 2004, AJ, 128, 2098

Springel, V. \& Hernquist, L. 2005, ApJ, 622, L9

Ueda, J., Iono, D., Yun, M. S., et al. 2014, ApJS, 214, 1

Wilson, C. D., Petitpas, G. R., Iono, D., et al. 2008, ApJS, 178, 189 